\title{
Targeting Biofilm of MDR Providencia stuartii by Phages Using a Catheter Model
}

\author{
Chani Rakov ${ }^{1}$, Shira Ben Porat ${ }^{1}$, Sivan Alkalay-Oren ${ }^{1}$, Ortal Yerushalmy ${ }^{1}$, Mohanad Abdalrhman \\ ${ }^{2}$, Niv Gronovich ${ }^{1}$, Lina Huang ${ }^{3}$, David Pride ${ }^{3}$, Shunit Coppenhagen-Glazer ${ }^{1}$, Ran Nir-Paz ${ }^{2}$ and \\ Ronen $\operatorname{Hazan}^{1 *}$ \\ ${ }^{1}$ Institute of Dental Sciences, School of Dentistry, Hebrew University of Jerusalem, Israel; \\ chani2061@gmail.com \\ ${ }^{2}$ Hadassah-Hebrew University Medical Center, Department of Clinical Microbiology and \\ Infectious Diseases, The faculty of medicine, Hebrew University of Jerusalem, Israel; \\ 3 Department of Pathology, University of California, San Diego, USA; \\ * Correspondence: ronenh@ekmd.huji.ac.il; Tel.: +97226758588
}

Received: date; Accepted: date; Published: date

Abstract: Providencia spp. are emerging pathogens mainly in nosocomial infections. Providencia stuartii in particular is involved in urinary tract infections and contributes significantly to the high incidence of biofilm-formation in catheterized patients. Furthermore, recent reports suggested a role for multiple drug resistant (MDR) P. stuartii in hospital-associated outbreaks which leads to excessive complications resulting in challenging treatments. Phage therapy is currently one of the most promising solutions to combat antibiotic-resistant infections. However, the number of available phages targeting Providencia spp. is extremely limited, restricting the use of phage therapy in such cases. In the present study, we describe the isolation and characterization of 17 lytic and lysogenic bacteriophages targeting clinical isolates of Providencia spp. as part of the Israeli Phage Bank (IPB). These phages, isolated from sewage samples, were evaluated for host range activity and effectively eradicated $95 \%$ of the tested bacterial strains isolated from different geographic locations and displaying a wide range of antibiotic resistance. Their lytic activity is demonstrated on agar plates, planktonic cultures, and biofilm formed in a catheter model. The results suggest that these bacteriophages can potentially be used for treatment of antibiotic-resistant Providencia spp. infections in general and of urinary tract infections in particular.

Keywords: phage therapy; antibiotic resistance; Providencia stuartii; Providencia rettgeri; catheter infections.

\section{Introduction}

Providencia stuartii and Providencia rettgeri are members of the Enterobacteriaceae family [1]. The involvement of these opportunistic pathogens in human health-care-associated infections was recognized long ago, and indeed several health care outbreaks of Providencia spp. have been reported $[2,3]$. While it is considered uncommon, recent reports from various countries established the emergence of multiple drug resistant (MDR) isolates of Providencia species [4-6] and demonstrated their ability to disseminate amongst patients in hospital settings leading to nosocomial outbreaks with treatment challenges and complications [7-9].

Various studies showed that Providencia species that are involved in urinary tract infections are also biofilm-forming $[10,11]$. The urinary tract provides a unique niche for the colonization of this bacterium [12]; therefore, it is highly prevalent in bacteriuric long-term-catheterized patients $[10,13]$. Infections by $P$. stuartii may reach mortality rates of $30 \%$ in nosocomial outbreaks [4]. Recent outbreaks of Providencia rettgeri have also raised public health concerns [7].

Providencia spp. harbor several different virulence factors such as chromosomal $a m p C$ gene and plasmid-mediated resistance mechanisms such as Extended-spectrum beta-lactamases (ESBLs) and Metallo-beta-lactamases (MBLs) [14]. Additionally, intrinsic inherited resistance to aminopenicillins, colistin, beta-lactams such as early-generation of cephalosporins, carbapenems, as well as aminoglycosides and many other antibiotic classes can also be found in this species[6,15-17]. Moreover, $P$. stuartii can create floating communities that facilitate adhesion and biofilm production 
leading to biofilm-associated infections [10]. These virulence factors make Providencia spp. difficult to treat [18].

Taking into account the increasing prevalence of Providencia spp. cases, their role in hospitalacquired infections, and the emergence of bacterial resistance, it is clear that additional or alternative tools for antibiotic treatments are necessary.

One such promising modality is phage therapy [19]. Indeed, the concept of harnessing bacteriophages to eradicate resistant infections has been re-discovered in western medicine and is gathering significant attention worldwide as a new tool to combat infectious diseases [20]. In the last few years, reports of compassionate-use cases and early-stage clinical trials are published, suggesting that bacteriophages against $P$. stuartii might also serve as a potential treatment. Nevertheless, to the best of our knowledge, the number of available Providencia spp. phages is rather limited [21] and their description and analysis is incomplete [22].

Here, we describe the isolation and characterization of 12 lytic and 5 lysogenic bacteriophages including full genome sequencing performed by the Israeli Phage Bank (IPB) [23]. The phages target multi-drug resistant clinical isolates of Providencia spp. across the world. We tested the treatment potential of these phages, by demonstrating their ability to eradicate biofilms built in a catheter model. As the phages present high efficiency in killing bacteria, we suggest that they might be useful as therapeutic agents.

\section{Materials and Methods}

\subsection{Bacterial Strains}

In this work, 41 clinical isolates of Providencia stuartii and Providencia rettgeri were used (Table 1). 10 of them were isolated locally, at the clinical microbiology lab, Hadassah-Hebrew University Medical Center, Jerusalem, Israel, and 31 strains were provided by the Center for Innovative Phage Applications and Therapeutics (IPATH) of the University of California, San Diego. Bacterial strains were grown in LB broth (Difco, Sparks, MD) at $37{ }^{\circ} \mathrm{C}$ shaken on a rotary shaker at $200 \mathrm{rpm}$ under aerobic conditions. Bacterial resistance or sensitivity to antibiotics was determined by the infectious disease unit of Hadassah Hospital, Jerusalem using VITEK (BioMérieux).

Table 1. Bacterial strains, their body site source, the provider of the isolates and antibiotic resistance.

\begin{tabular}{|c|c|c|c|}
\hline Bacterial Strains & Source & Provided by ${ }^{1}$ & Antibiotic Resistance ${ }^{2}$ \\
\hline \multicolumn{4}{|l|}{ Providencia stuartii } \\
\hline P.st 1 & blood & $H M C$ & $\begin{array}{l}\text { AMP, SAM, CRO, } \\
\text { CXM, GEN, CFZ }\end{array}$ \\
\hline P.st 2 & blood & $H M C$ & $\begin{array}{l}\text { AMP, SAM, CIP, CRO, } \\
\text { CXM, GEN, CFZ }\end{array}$ \\
\hline P.st 3 & blood & $H M C$ & $\begin{array}{l}\text { AMP, SAM, CIP, AMK, } \\
\text { CRO, CXM, GEN, CFZ }\end{array}$ \\
\hline P.st 4 & blood & $H M C$ & $\begin{array}{l}\text { AMP, SAM, CRO, } \\
\text { CXM, GEN, CFZ }\end{array}$ \\
\hline P.st 5 & blood & $H M C$ & $\begin{array}{l}\text { AMP, SAM, CIP, AMK, } \\
\text { CRO, CXM, GEN, CFZ }\end{array}$ \\
\hline P.st 6 & blood & $H M C$ & $\begin{array}{l}\text { AMP, SAM, CIP, AMK, } \\
\text { CRO, CXM, GEN, CFZ }\end{array}$ \\
\hline P.st 7 & blood & $H M C$ & $\begin{array}{c}\text { AMP, SAM, CIP, CXM, } \\
\text { GEN, CFZ }\end{array}$ \\
\hline P.st 8 & blood & $H M C$ & $\begin{array}{l}\text { AMP, SAM, CIP, AMK, } \\
\text { CXM, CRO, GEN, CFZ }\end{array}$ \\
\hline P.st 9 & blood & $H M C$ & AMP, GEN \\
\hline PR02 & urine & $U C S D$ & $\begin{array}{l}\text { AMP, SAM, CXM, } \\
\text { GEN, CFZ }\end{array}$ \\
\hline PR03 & urine & $U C S D$ & $\begin{array}{l}\text { AMP, SAM, CXM, } \\
\text { GEN, CFZ }\end{array}$ \\
\hline
\end{tabular}




\begin{tabular}{|c|c|c|c|}
\hline PR04 & urine & $U C S D$ & $\begin{array}{c}\text { AMP, SAM, AMK, } \\
\text { CXM, GEN, CFZ }\end{array}$ \\
\hline PR12 & leg lesion & $U C S D$ & AMP, SAM, CIP, CFZ \\
\hline PR13 & leg lesion & $U C S D$ & AMP, SAM, CRO, CFZ \\
\hline PR14 & urine & $U C S D$ & AMP, SAM, CFZ \\
\hline PR15 & blood & $U C S D$ & AMP, SAM, CIP \\
\hline PR16 & urine & $U C S D$ & \\
\hline PR25 & blood & $U C S D$ & AMP, CXM, GEN \\
\hline PR26 & blood & $U C S D$ & AMP, CXM, GEN \\
\hline PR32 & urine catheter & $U C S D$ & AMP, GEN, CFZ \\
\hline \multicolumn{4}{|l|}{$\begin{array}{l}\text { Providencia } \\
\text { rettgeri }\end{array}$} \\
\hline P.rett10 & blood & $H M C$ & AMP, GEN \\
\hline PR01 & breast & $U C S D$ & AMP, SAM, GEN, CFZ \\
\hline PR06 & blood & $U C S D$ & SAM, CFZ \\
\hline PR07 & urine & $U C S D$ & AMP, SAM, CFZ \\
\hline PR08 & sputum & $U C S D$ & AMP, SAM, CFZ \\
\hline PR09 & urine & $U C S D$ & AMP, SAM, CRO, CFZ \\
\hline PR10 & heel lesion & $U C S D$ & AMP, SAM, CIP, CFZ \\
\hline PR11 & lesion & $U C S D$ & AMP, SAM, CFZ \\
\hline PR17 & foot culture & $U C S D$ & AMP, SAM \\
\hline PR18 & urine & $U C S D$ & AMP, SAM \\
\hline PR19 & urine & $U C S D$ & AMP, GEN \\
\hline PR20 & body site, sacrum & $U C S D$ & \\
\hline PR21 & body tissue, foot & $U C S D$ & AMP, GEN \\
\hline PR22 & urine & $U C S D$ & AMP, SAM, GEN \\
\hline PR23 & urine & $U C S D$ & AMP, GEN \\
\hline PR24 & urine & $U C S D$ & AMP, GEN \\
\hline PR27 & body site, bladder stone & $U C S D$ & AMP, CXM, GEN \\
\hline PR28 & blood & $U C S D$ & AMP, CXM, GEN \\
\hline PR29 & urine & $U C S D$ & AMP, CXM, GEN \\
\hline PR30 & sputum & $U C S D$ & AMP, GEN \\
\hline PR31 & nasopharyngeal & $U C S D$ & AMP, GEN \\
\hline
\end{tabular}

${ }^{1}$ HMC stands for Hadassah Medical center, and UCSD stands for University of California San Diego.

${ }^{2}$ The antibiotics names are given in the table as abbreviations: AMP stands for ampicillin, SAM for ampicillin/sulbactam, CIP for ciprofloxacin, MEM for meropenem, ETP for ertapenem, GEN for gentamicin, CXM for ceftriaxone, CRO for cefuroxime, AMK for amikacin, and CFZ for cefazolin

\subsection{Phage Isolation and propagation}

Isolation of phages was performed using the standard double-layer agar method previously described [24] with mild modifications. Preliminary enrichment of phages was carried out by overnight growth of the bacteria in an environmental sewage sample collected from various places in Jerusalem and the surrounding. Samples were centrifuged at $7800 \mathrm{rpm}$ for $10 \mathrm{~min}$ (centrifuge 5430R, rotor FA45-24-11HS; Eppendorf). The supernatant was filtered through $0.22-\mu \mathrm{m}$-pore-size filters (Merck Millipore, Ltd., Ireland) and LB 5X was then added to the filtered sewage. Grown bacterial cultures of Providencia stuartii and Providencia rettgeri were mixed and inoculated with filtered sewage and LB overnight at $37^{\circ} \mathrm{C}$ shaken on a rotary shaker at $200 \mathrm{rpm}$ under aerobic conditions. Following inoculation, the liquid was centrifuged and re-filtered through $0.22-\mu \mathrm{m}$-pore-size filters. The filtered lysate was then spotted on double-layered agarose plates which were incubated overnight at $37{ }^{\circ} \mathrm{C}$. Double-layered agarose plates were prepared by covering LB plates with $3.5 \mathrm{ml}$ of pre-warmed agarose $(0.25 \%)$ containing $0.1 \mathrm{ml}$ of an overnight culture of bacteria (Table 1). Clear plaques were observed and purified by minimum four passages (of repeatedly picking single plaques and spreading them on a new bacterial lawn). Finally, single plaques were collected and transferred into bacterial liquid cultures to 
was determined by counting plaque-forming units (PFU) as described [24]. Briefly, the lysate was serially diluted 10 -fold into LB broth, and drops of $5 \mathrm{ul}$ were spotted on overlaid tested bacterial strain lawns of agarose $(0.25 \%)$, and then incubated overnight at $37^{\circ} \mathrm{C}$. The plaques were counted, and the initial concentration of $\mathrm{PFU} / \mathrm{mL}$ was calculated.

\subsection{Phgae Genome Sequencing}

The phages' DNA was purified as described [25] using a phage DNA isolation kit (Norgen Biotek), and libraries were prepared with a Nextera XT DNA kit (catalog number FC-131-1096; Illumina, San Diego, CA). Normalization, pooling, and tagging were performed in a common flow cell with $1 \times 150$ bp paired-end reads, which were used for sequencing with the Illumina NextSeq 500 platform. Sequencing was performed in the sequencing unit of The Hebrew University of Jerusalem at the Hadassah Campus. Trimming, quality control, assembly of reads, and analyses were performed using Geneious Prime 2020 and its plugins (https://www.geneious.com). Assembly was commonly performed using the SPAdes plugin of Geneious Prime 2020. Annotation was performed using RAST (https://rast.nmpdr.org/rast.cgi), FASTER (https://phaster.ca), and BLAST servers.

\subsection{Bacteriophages for phylogenetic tree}

The genomes of published phages used for comparison are listed in Table 2.

Table 2. Published genomes

\begin{tabular}{ccc}
\hline Type & Phage name & Accession number \\
\hline Providencia phage & Redjac & NC_018832.1 \\
Providencia phage (P. stuartii) & vB PstP PS3 & NC_048148.1 \\
Providencia phage (P. stuartii) & vB_PstP_Stuart & MK387869.1 \\
Providencia phage (P. rettgeri) & vB_PreS_PR1 & NC_041913.1 \\
Providencia phage (P. rettgeri) & vB_PreS-PatoteraRojo & MT675126.1 \\
Providencia phage (P. rettgeri) & vB_PreS-Stilesk & MT675125.1 \\
Providencia phage (P. rettgeri) & vB_PreS-PibeRecoleta & MT675124.1 \\
Morganella phage & MmP1 & NC_011085.3 \\
Morganella phage & vB_MmoM_MP1 & NC_031020.1 \\
Proteus phage & pPM_01 & NC_028812.1 \\
Shigella phage & vB_SdyM_006 & MK295204.1 \\
Siphoviridae sp. isolate 404 & - & MN855780.1
\end{tabular}

\subsection{TEM visualization}

To visualize the geometric structure of the phages, transmission electron microscopy (TEM) was used as described in OpenWetWare (http://openwetware.org/wiki /Gill: Preparingphage_specimens_for_TEM). Two $\mathrm{ml}$ of phage lysate with $10^{9} \mathrm{PFU} / \mathrm{ml}$ were centrifuged at 14,000 rpm (centrifuge 5430R, rotor FA-45-24-11HS; Eppendorf) for $2 \mathrm{~h}$ at room temperature. The pellet was resuspended in $200 \mu \mathrm{l}$ of $5 \mathrm{mM} \mathrm{MgSO} 4$ and incubated overnight at $4^{\circ} \mathrm{C}$. For visualizing the phage-bacteria interactions $1 \mathrm{ml}$ of lysate with $10^{8} \mathrm{PFU} / \mathrm{ml}$ was inoculated with $100 \mathrm{ul}$ of overnight grown bacteria for $15 \mathrm{~min}$. Then, $100 \mathrm{ul}$ of the bacteria and phage mix were transferred into $100 \mathrm{ul}$ of 5 $\mathrm{mM} \mathrm{MgSO} 4$. On a strip of parafilm, $30 \mathrm{ul}$ of $5 \mathrm{mM} \mathrm{MgSO} 4$ and $10 \mathrm{ul}$ of the phage sample were mixed gently. Grids were prepared by placing on the drop of phage sample with the carbon side facing down for a minute, and then on the drop of the stain ( $2 \%$ uranyl acetate) for another minute. After drying, a transmission electron microscope (Joel, TEM 1400 plus) with a charge-coupled device camera (Gatan Orius 600) was used to capture images.

\subsection{Assessment of phage lytic activity in planktonic cultures}

Lytic activity of the phage was assessed by inoculation of logarithmic or stationary Providencia cultures with phages in triplicates. The growth and lysis kinetics were recorded by measuring the optical density at a wavelength of $600 \mathrm{~nm}(\mathrm{OD} 600 \mathrm{~nm})$ at $37^{\circ} \mathrm{C}$ with 5-s shaking every 20 min using a 96-well plate reader (Synergy; BioTek, Winooski, VT)

\subsection{Assessment of phage lytic activity in biofilm}


$100 \%$ Latex (silicon coated) or silicone catheters (Foley Catheter) at a thickness of 16Fr/Ch and volume of 5-15ml were cut into ring-like pieces of $\sim 2-3 \mathrm{~mm}$ width. The catheter pieces were placed in $15 \mathrm{ml}$ tubes containing $3 \mathrm{ml} \mathrm{LB}$ broth with $100 \mathrm{ul}$ of an overnight culture of bacteria. Biofilms were grown for 4 days at $37^{\circ} \mathrm{C}$ shaken on a rotary shaker at $200 \mathrm{rpm}$ under aerobic conditions. After transferring the catheter to a new tube and inoculation of the phage, incubation was continued overnight. Biofilms built on latex catheters were infected with PSTCR4 phage and biofilms built on silicone catheters were infected with a cocktail of PSTCR4 and PSTCR6 at the same concentration.

The catheter pieces were washed with phosphate-buffered saline (PBS), and the biomass was quantified using crystal violet staining as previously described [26]. Briefly, fixation was achieved by soaking the catheter in methanol, followed by incubation for $20 \mathrm{~min}$, followed in turn by methanol aspiration and air drying. The biofilms were stained by crystal violet (1\%) for 20 min at room temperature and then washed with water. The stain was extracted by adding ethanol (100\%) and biomass was quantified by determining the optical density at $538 \mathrm{~nm}$ (OD538). In addition, the biofilm on the catheter was stained using the live stain (syto) of the Live/Dead cell viability kits (Life Technologies, Waltham, MA) according to the manufacturer's instructions. The fluorescence emission of the biofilms was detected using a confocal laser microscope (Nikon Yokogaha W1 spinning disk). The microscopy slices were combined to a 3D image using NIS program. For viable count, each catheter piece was transferred to $1 \mathrm{ml}$ of water in a $1.5 \mathrm{~mL}$ tubes and placed in a sonicating water bath (Bandelin Sonopuls HD 2200, Berlin, Germany) for $7 \mathrm{~min}$ to disrupt the biofilm, and $10 \mu \mathrm{L}$ of 10 -fold serial dilutions of each sample were plated on LB agar plates. Colonies were counted after $24 \mathrm{~h}$ at $37 \circ \mathrm{C}$ and the number of colony-forming units $(\mathrm{CFU}) / \mathrm{ml}$ was calculated.

\subsection{Statistical Analysis}

GraphPad prism 8.0.2 (263) was used for the statistical analysis, graph formation, and design. The results were analyzed as the mean \pm standard deviation in each experimental group. Statistical significance was calculated by a Student's $t$-test two-tailed unpaired $p$ values (significance level: $p<$ $0.05)$.

\section{Results}

\subsection{Phage Isolation and Assessment of Host-Range coverage}

A screening of sewage samples for the presence of potential anti-Providencia species bacteriophages was performed as described in "Materials and Methods" (phage isolation and propagation). After a preliminary enrichment step, and subsequent spotting of the lysate on bacterial lawns, numerous samples resulted in reproducible lysis of bacterial cells and exhibited clear plaques. 17 phages were isolated from single plaques (Table 3) and further propagated in liquid culture as detailed in "Materials and Methods" showing lysis after an overnight incubation in liquid culture. The phages were evaluated for host range activity by the drop test. As the goal here was to supply a satisfying coverage of Providencia spp., strains that showed sensitivity to one of the phages were excluded from the subsequent screening cycles. The results demonstrated that despite the bacteria's wide-range antibiotic resistance, the phages targeted 39 out of 41 clinical isolates, providing a coverage of $95 \%$ of the isolates tested here (Figure 1).

\subsection{Genome Analysis}

Bacteriophages' genomes were subsequently sequenced and annotated. Phages' names, their putative lifecycle type, topology, genome size, phylogeny, and GenBank accession number are denoted in Table 3.

Table 3. Phage collection

\begin{tabular}{cccccc}
\hline phage & $\begin{array}{c}\text { Putative } \\
\text { Life-cycle } \\
\text { Type }\end{array}$ & Topology & $\begin{array}{c}\text { Genome } \\
\text { Size } \\
\text { (bp) }\end{array}$ & Phylogeny & $\begin{array}{c}\text { GenBank } \\
\text { Accession }\end{array}$ \\
\hline Kokobel1 & lytic & circular & 59,837 & $\begin{array}{c}\text { Caudovirales; } \\
\text { Siphoridae. }\end{array}$ & MW145139.1 \\
\hline
\end{tabular}


bioRxiv preprint doi: https://doi org/101101/2021.03.02 433687; this version posted March 3,2021 . The copyright holder for this preprint (which was not certified by peer review) is the author/funder, who has granted bioRxiv a license to display the preprint in perpetuity. It is made available under aCC-BY-NC-ND 4.0 International license.

\begin{tabular}{|c|c|c|c|c|c|}
\hline Kokobel2 & lytic & circular & 45,880 & $\begin{array}{l}\text { Caudovirales; } \\
\text { Siphoridae }\end{array}$ & MW145138.1 \\
\hline PSTNGR2lys & lysogenic & circular & 50,958 & Caudovirales; & MW145137.1 \\
\hline PSTCR2 & lytic & circular & 40,200 & $\begin{array}{c}\text { Siphoridae } \\
\text { unclassified } \\
\text { Studiervirinae } \\
\text {,Autographiviridae } \\
\text { family }\end{array}$ & MW057854.1 \\
\hline PSTCR3 & lysogenic & circular & 39,447 & Caudovirales; & MW057855.1 \\
\hline PSTCR4 & lytic & circular & 57,214 & $\begin{array}{l}\text { Myoviridae } \\
\text { Caudovirales; } \\
\text { Siphoridae }\end{array}$ & MW057856.1 \\
\hline PSTCR5 & lytic & circular & 109,434 & $\begin{array}{l}\text { Caudovirales; } \\
\text { Siphoridae; } \\
\text { Priunavirus }\end{array}$ & MW057857.1 \\
\hline PSTCR6 & lytic & circular & 155,737 & $\begin{array}{c}\text { Tevenvirinae; } \\
\text { unclassified } \\
\text { Tevenvirinae }\end{array}$ & MW057858.1 \\
\hline PSTCR7 & lytic & circular & 57,986 & $\begin{array}{c}\text { Caudovirales; } \\
\text { Siphoviridae }\end{array}$ & MW057861.1 \\
\hline PSTCR7lys & $\begin{array}{l}\text { lysogenic- } \\
\text { partial } \\
\text { genome }\end{array}$ & linear & 22,938 & $\begin{array}{l}\text { Caudovirales; } \\
\text { Siphoridae }\end{array}$ & MW057862.1 \\
\hline PSTCR8lys & lysogenic & linear & 40,280 & $\begin{array}{l}\text { Caudovirales; } \\
\text { Siphoridae }\end{array}$ & MW057859.1 \\
\hline PSTCR9 & lytic & circular & 58,873 & $\begin{array}{c}\text { Caudovirales; } \\
\text { Siphoviridae }\end{array}$ & MW057860.1 \\
\hline PSTRCR_114 & lytic & circular & 42,750 & $\begin{array}{c}\text { Autographiviridae; } \\
\text { Kakivirus }\end{array}$ & MW358930.1 \\
\hline PSTRCR_117lys & lysogenic & linear & 41,059 & $\begin{array}{c}\text { Caudovirales; } \\
\text { Siphoviridae }\end{array}$ & MW358929.1 \\
\hline PSTRCR_120 & lytic & circular & 39,396 & $\begin{array}{c}\text { Autographiviridae; } \\
\text { unclassified } \\
\text { Studiervirinae }\end{array}$ & MW358928.1 \\
\hline PSTRCR_121 & lytic & circular & 165,776 & $\begin{array}{l}\text { Myoviridae; } \\
\text { unclassified } \\
\text { Tevenvirinae }\end{array}$ & $\underline{\text { MW385300.1 }}$ \\
\hline PSTRCR_127 & lytic & circular & 166,815 & $\begin{array}{l}\text { Myoviridae; } \\
\text { unclassified } \\
\text { Tevenvirinae }\end{array}$ & $\underline{\text { MW358927.1 }}$ \\
\hline
\end{tabular}

${ }^{1}$ The determination of lytic/ lysogenic is based on BLAST database. In cases where the full genome analysis of the phage revealed that it is a part of a bacterial genome it is an indication of lysogeny. Another indication for lysogeny is the similarities of the phage genes to elements involved in lysogenicity such as a lambda CI repressor, integrase, or transposon-like elements. 
Alignment of the genomes revealed a high diversity between the phages reported here. According to NCBI database, the lytic phages PSTCR4, PSTCR6, PSTCR7 and Kokobel2 do not have high similarity to known phages. PSTCR4 and PSTCR7 do not overlap any other published genomes $(0 \%$ query cover), those two phages have high similarity to each other with $93 \%$ coverage, $97 \%$ identity, and about 9\% coverage with Kokobel2. PSTCR6 have only $20 \%$ coverage with the known Shigella phage vB_SdyM_006 (MK295204.1). The lysogenic phage PSTCR7lys which has some similarities with regions inside known Providencia strains was found to be unique and it seems that it belongs to a new phage subfamily. Some of the phages have genomic similarity to phages with different hosts; Kokobel1 genome displays similarity to Proteus phages and PSTRCR_121, PSTRCR_127 and PSRCR_128 display similarity to Morganella phages. The phylogenetic tree of our phages compared with the few published Providencia phages and other relevant phages (obtained from the NCBI database) are provided in Figure 2.

As potential candidates for phage therapy treatment, the phages were also scanned for virulence factors and antimicrobial resistance genes using Abricate (https://github.com/tseemann/abricate) with all its databases: NCBI, CARD, ARG-ANNOT, Resfinder, EcOH, MEGARES, PlasmidFinder, Ecoli_VF and VFDB. The phages were found to be free of virulence factors and antibiotic resistance genes (data not shown).

\subsection{Characterization of phage lytic activity}

To further test the phages' applicability against Providencia, lytic activity was assessed on agar plates, planktonic cultures, and biofilm formed on a catheter. The isolate P.st 8 (with its phages PSTCR4 and PSTCR6), was chosen to represent $P$. stuartii isolates for deeper investigation, since this bacterium was found to be one of the most resistant isolates ( 8 out of 10 antibiotics, see table 1 in "Materials and Methods"). P.rett10 (with its phage PSTCR2), was selected arbitrarily, to represent $P$. rettgeri isolates.

Consistent with the fact that these phages were confirmed to be genotypically distinct from each other, the infectivity patterns of the different phages were found to be also unique phenotypically: bacteriophages (PSTCR4, PSTCR6, and PSTCR2) formed plaques of different sizes and transparencies levels, and some of them had a halo region surrounding the plaque (Figure $3 \mathrm{a}-3 \mathrm{c}$ ).

\subsection{Electron microscopy visualizing}

To observe and determine the geometric structure and morphological characteristics of PSTCR4, PSTCR6 and PSTCR2, these phages were visualized under Transmission Electron Microscope (TEM). TEM microscopy showed that PSTCR4 has a hexagonal capsid with a measured diameter of $105 / 75 \mathrm{~nm}$ and a tail length of $61 \mathrm{~nm}$ with long fibers (Figure 3d). PSTCR6 has a hexagonal capsid with a measured diameter of 101/66 nm and a tail length of $60 \mathrm{~nm}$ with long-tailed fibers. In most images obtained, phages were attached or were hidden between vesicles secreted by the bacteria. (Figure 3e). PSTCR2 has a hexagonal capsid with a measured diameter of 55/50 nm and a very short tail length of $18 \mathrm{~nm}$ (Figure 3f).

Phages also exhibited different inhibition patterns, as observed by the different kinetics of bacterial growth curves in the presence of the different phages: At the P. stuartii logarithmic stage, while treatment of PSCR 4 showed an effect on bacterial growth only after 10 hours with the best lysis after 23 hours followed by a regrowth of the bacteria, PSTCR6 kept bacteria from growing for the first 10 hours, followed by a regrowth that was disrupted by another lysis peak towards the stationary stage (Figure 4a). Cultures treated with both phages (PSTCR4 and PSTCR6) displayed a synergistic effect and kept bacteria from growing throughout 50 hours of the experiment (Figure 4a). At the stationary phase of P.st 8, PSTCR4 exhibited a slight lytic effect after 40 hours of growth, while PSTCR6 acted earlier, after 27 hours, and no additive effect was obtained when both phages were added (Figure 4b). Impressive actual lysis of P. rettgeri cultures was observed by PSTCR2 totally inhibiting logarithmic bacterial growth throughout the time of the experiment (Figure 4c) and reducing stationary culture turbidity 2.4 -fold (Figure $4 \mathrm{~d}$ ). 
bioRxiv preprint doi: https://doi.org/10.1101/2021.03.02.433687; this version posted March 3, 2021. The copyright holder for this preprint (which was not certified by peer review) is the author/funder, who has granted bioRxiv a license to display the preprint in perpetuity. It is made available under aCC-BY-NC-ND 4.0 International license.

\subsection{Biofilm degredation activity}

Biofilm infections pose a huge challenge to human health as they are very difficult to treat by most antibacterial strategies, including conventional antibiotics [27]. Thus, an assessment of phages' efficacy against $P$. stuartii biofilm formed on a catheter was performed using one of the most resistant isolates, P.st 8 and its 2 lytic phages (PSTCR4 and PSTCR6) as a representative model. Latex and silicone catheters were cut to ring-shaped pieces and infected with bacteria at the logarithmic stage, once the biofilm was established, phage was inoculated and incubated overnight. The cultures which contained phage were less turbid compared to the cultures without phage as can be seen in Figure 5a.

The biofilm degradation activity was assessed by biomass staining, Viable count of bacteria, SEM and confocal microscopy as described in detail in "materials and methods". Crystal violet biofilm biomass evaluation showed a 1.9- and 2-fold reduction of 3-day-old $P$. stuartii biofilms built on latex or silicone catheters respectively, following 24 hours of treatment, in comparison with the untreated biofilm controls (Figure 5b). To exclude the interference of bacterial debris and to verify the eradication of live bacteria, we further tested the viable counts of the biofilm itself. Biofilms were disrupted by sonication and viable counts of live bacteria were performed. Viable counts showed a 2.86- and 2.46-log growth reduction by phage exposure in latex and silicone catheters respectively, compared to untreated biofilms (Figure 5c).

To further assess phage efficacy against $P$. stuartii biofilm, SEM was used to visualize the effect of phage on a 3-day-old biofilm. Figure 5d shows the surface topography of naked latex and silicone catheters and biofilms formed on those catheters. The surface area of the latex catheter appeared to have protrusions and spherical structures. Exposure to phage revealed an extensive effect of bacterial lysis leaving cellular debris and almost no trace of the biofilm. (Figure 5d).

Finally, catheters were stained with the fluorescence stain syto and visualized by a confocal laser microscope. A significant difference was observed in biofilm formed on a catheter treated with phage compared with the untreated controls (Figure 5e). This method was not appropiate for the latex catheters as for an unknown reason, the catheter itself absorbs the stain more efficiently than the biofilm treated with phage (Figure 5e).

\section{Discussion}

This article reports the isolation, characterization, and full genome sequencing of 17 novel phages targeting $P$. stuartii and $P$. rettgeri clinical isolates, providing a satisfying coverage of $95 \%$ of the host strains tested. The phages were active against Providencia isolates across the world, portending for their activity against yet unknown strains.

Some of the phages' genomes were found to be unique exhibiting distinct differences compared to each other and compared to other known genomes in the NCBI databases. In our experience, this is rather unusual in comparison to other phage analyses we previously conducted [23].

The extreme specificity of phages requires a personalized-medicine approach. Therefore, characterization of all 17 phages with all 41 bacterial hosts is tedious and irrelevant. When a candidate patient is ready for treatment, we screen all our phages to assess their killing abilities and characterize the most suitable one on the patient's specific isolate. Therefore here, characterization has been carried out on representative phage and hosts. In-vitro characterization of 3 of the lytic phages (PSTCR2, PSTCR4 and PSTCR6) showed killing capabilities in solid and liquid cultures in various patterns and levels of efficiencies.

Consistent with the results for the planktonic cultures, the phages PSTCR4 and PSTCR6 exhibited an efficient reduction of well-established $P$. stuartii biofilms formed in catheter models using various methods. These results, together with the finding that the phages are free of virulence factors and resistance genes, suggest the potential of those candidate phages as reliable therapeutic agents for phage therapy against $P$. stuartii biofilms in bacteriuric catheterized patients.

Nevertheless, as the ability to disrupt biofilms is not obvious for any phage, we postulate that the phages' ability to remove biofilm should also be tested by adding small volumes of phage to the stationary culture in which the biofilm is formed, or alternatively to dialyze the phage suspension 
before inoculation. This is due to the possibility that additional nutrients that remain in the phage media might trigger bacteria in a biofilm state to switch to logarithmic growth. These circumstances might accentuate the killing effect of most phages.

Further steps should be taken to promote phage therapy against biofilms. These include: developing fast and accurate techniques for the prediction and matching of phages against biofilms, and establishing a large collection of anti-biofilm specialized phages by selective enrichment. Such improvement might have extensive clinical implications.

Though Providencia spp. are not defined yet as part of the ESCAPE group of challenging antibiotic resistant pathogens [28], the overuse of wide-spectrum antibiotics may result in the emergence of extreme resistance patterns and lead to more nosocomial outbreaks, which explains the growing concern among health professionals.

Currently, there are not enough available effective options to fight cases of Providencia biofilms infection: an extremely limited number of phages targeting $P$. stuartii and $P$. rettgeri were isolated, and no phage therapy was applied using Providencia phages. Thus, the results presented here open new avenues for bacteriuric long-term catheterized patients, and for the phage therapy field.

In conclusion, our results indicate that though resistant to antibiotics, Providencia strains might be sensitive to phages, a promising direction to consider in the continuous struggle against the current emergence of multidrug resistant Providencia spp.

\section{Figure Legends}

Figure 1. Reduction screen. The bacterial isolates (Y axis) killed by each noted phage (X axis) are presented in green, and those that remained for the next screen are in red after phage exposure. First all bacterial strains were screened by the first phage. Then the resistant bacteria were screen iteratively by the second phage and so on and so forth. At the end, only two bacterial strains reamined resistant to all available phages.

Figure 2. Genome analysis of Providencia phages. Phylogenetic tree of Providencia phages. Phages of this work are shown in red. Close-similar genomes of fully sequenced phages from the NCBI database are given as reference points shown in black.

Figure 3. Plaque morphologies and TEM microscopy of anti-Providencia phages. Single plaques of PSTCR4 (a), PSTCR6 (b) on P.st 8, and PSTCR2 (c) on P.rett10 on bacterial lawns. Geometric structure of PSTCR4 (d); PSTCR6(e); and PSTCR2 attached to its host P.rett10 (f).

Figure 4. Characterization of lytic activity in liquid cultures. Kinetic growth curves of P.st 8 (blue) at the (a) logarithmic and (b) stationary stage in the presence of the phages PSTCR4 (red), PSTCR6 (green) and both of them (purple); Growth curves of P.rett10 (blue) at the logarithmic (c) and stationary (d) stage with the presence of the phage PSTCR2 (red).

Figure 5. Biofilm degradation activity. (a) Model of $P$. stuartii biofilm formed on Latex and Silicone catheters in the absence (left tube) and presence (right tube) of phage. (b) Biofilm biomass quantification by crystal violet staining of $P$. stuartii formed on latex and silicone catheters; (c) Viable CFU counts of disrupted P. stuartii biofilms formed on latex and silicone catheters. (d) Scanning Electron Microscopy (SEM) of untreated, P.st 8 biofilm infected and phage-treated latex and silicone catheter surfaces; (e) Confocal microscopy of untreated, P.st 8 biofilm infected and phage-treated latex and silicone catheter biofilms.

\section{Acknowledgments.}

The authors would like to thank the "United States - Israel Binational Science Foundation (BSF)" grant \#2017123, the Israel Science Foundation (ISF) IPMP grant \#ISF1349/20, the Rosetrees Trust grant A2232, and the Milgrom Family Support Program. We would like also to thank the dedicated team of the Facility Core of Hadassah Campus of the Hebrew University for their help in sequencing and electron microscopy and Hanan Levi from the 

samples.

\section{References}

1. O'Hara, C.M.; Brenner, F.W.; Miller, J.M. Classification, identification, and clinical significance of Proteus, Providencia, and Morganella. Clin. Microbiol. Rev. 2000, 13, 534 546.

2. JA, W.; DH, S.; A, H.; AH, S.; WJ, M. Nosocomially Acquired Bacteriuria Due to Proteus

3. JW, W. Providencia Stuartii: A Common Cause of Antibiotic-Resistant Bacteriuria in Patients

4. Choi, H.K.; Kim, Y.K.; Kim, H.Y.; Park, J.E.; Uh, Y. Clinical and microbiological features of providencia bacteremia: Experience at a tertiary care hospital. Korean J. Intern. Med. 2015, 30, 219-225, doi:10.3904/kjim.2015.30.2.219.

5. Molnár, S.; Flonta, M.M.M.; Almaş, A.; Buzea, M.; Licker, M.; Rus, M.; Földes, A.; Székely,

6. Hayakawa, K.; Marchaim, D.; Divine, G.W.; Pogue, J.M.; Kumar, S.; Lephart, P.; Risko, K.; E. Dissemination of NDM-1 carbapenemase-producer Providencia stuartii strains in Romanian hospitals: a multicentre study. J. Hosp. Infect. 2019, 103, 165-169, doi:10.1016/j.jhin.2019.04.015.

7. S, S.; N, N.; J, D. Providencia Rettgeri: An Emerging Nosocomial Uropathogen in an

8. Zavascki, A.P.; Carvalhaes, C.G.; da Silva, G.L.; Soares, S.P.T.; de Alcåntara, L.R.; Elias, Sobel, J.D.; Kaye, K.S. Growing prevalence of Providencia stuartii associated with the increased usage of colistin at a tertiary health care center. Int. J. Infect. Dis. 2012, 16. L.S.; Sandri, A.M.; Gales, A.C. Outbreak of Carbapenem-Resistant Providencia stuartii in an Intensive Care Unit . Infect. Control Hosp. Epidemiol. 2012, 33, 627-630, doi:10.1086/665730. S.; Horhat, F.G.; Baditoiu, L. Distribution of NDM1 carbapenemase-producing proteeae 
strains on high-risk hospital wards. Infect. Drug Resist. 2020, 13, 4751-4761, doi:10.2147/IDR.S280977.

10. El Khatib, M.; Tran, Q.T.; Nasrallah, C.; Lopes, J.; Bolla, J.M.; Vivaudou, M.; Pagès, J.M.;

11. Stickler, D.J. Bacterial biofilms in patients with indwelling urinary catheters. Nat. Clin. Pract. Urol. 2008, 5, 598-608.

12. Fletcher, M.; Oppenheimer, S.R.; Warren, J.W. Colonization of urinary catheters by Escherichia coli and providencia stuartii in a laboratory model system. In Proceedings of the Journal of Urology; Elsevier Inc., 1994; Vol. 152, pp. 232-236.

13. Fierer, J.; Ekstrom, M. An Outbreak of Providencia stuartii Urinary Tract Infections: Patients 1553-1555, doi:10.1001/jama.1981.03310400035023.

14. Tran, Q.T.; Mahendran, K.R.; Hajjar, E.; Ceccarelli, M.; Davin-Regli, A.; Winterhalter, M.; Weingart, H.; Pagès, J.M. Implication of porins in $\beta$-lactam resistance of Providencia stuartii. J. Biol. Chem. 2010, 285, 32273-32281, doi:10.1074/jbc.M110.143305.

15. Stock, I.; Wiedemann, B. Natural antibiotic susceptibility of providencia stuartii, P. rettgeri, P. alcalifaciens and P. rustigianii strains. J. Med. Microbiol. 1998, 47, 629-642, doi:10.1099/00222615-47-7-629.

16. McHale, P.J.; Keane, C.T.; Dougan, G. Antibiotic resistance in Providencia stuartii isolated

17. Tshisevhe, V.S.; Lekalakala, M.R.; Tshuma, N.; Janse van Rensburg, S.; Mbelle, N. Outbreak

18. N, K.; V, V.; M, S.; T, E.; A, M. The Potential Virulence Factors of Providencia stuartii:

19. Lin, D.M.; Koskella, B.; Lin, H.C. Phage therapy: An alternative to antibiotics in the age of multi-drug resistance. World J. Gastrointest. Pharmacol. Ther. 2017, 8, 162, doi:10.4292/wjgpt.v8.i3.162.

20. Hanlon, G.W. Bacteriophages: an appraisal of their role in the treatment of bacterial infections. Int. J. Antimicrob. Agents 2007, 30, 118-128.

21. Oliveira, H.; Pinto, G.; Hendrix, H.; Noben, J.P.; Gawor, J.; Kropinski, A.M.; Łobocka, M.; 
bioRxiv preprint doi: https//doi.org/10.1101/2021.03.02 433687· this version posted March 3,2021 . The copyright holder for this preprint (which was not certified by peer review) is the author/funder, who has granted bioRxiv a license to display the preprint in perpetuity. It is made available under aCC-BY-NC-ND 4.0 International license.

22. Oliveira, H.; Pinto, G.; Mendes, B.; Dias, O.; Hendrix, H.; Akturk, E.; Noben, J.P.; Gawor, J.; Łobocka, M.; Lavigne, R.; et al. A tailspike with exopolysaccharide depolymerase activity from a new Providencia stuartii phage makes multidrug-resistant bacteria susceptible to serum-mediated killing. Appl. Environ. Microbiol. 2020, 86, doi:10.1128/AEM.00073-20.

23. Yerushalmy, O.; Khalifa, L.; Gold, N.; Rakov, C.; Alkalay-Oren, S.; Adler, K.; Ben-Porat, S.; Kraitman, R.; Gronovich, N.; Ginat, K.S.; et al. The israeli phage bank (IPB). Antibiotics 2020, 9, doi:10.3390/antibiotics9050269.

24. Clokie, M.R.J.; Kropinski, A.M. Bacteriophages : methods and protocols Volume 1: Isolation, Characterization, and Interactions; 2009; ISBN 9781588296825 (hbk.)\$ \$r1588296822 (hbk.).

25. Clokie, M.R.J.; Kropinski, A.M. Bacteriophages Methods and Protocols Volume 2: Molecular and Applied Aspects; 2009; ISBN 978-1-60327-564-4.

26. Stepanović, S.; Vuković, D.; Dakić, I.; Savić, B.; Švabić-Vlahović, M. A modified microtiterplate test for quantification of staphylococcal biofilm formation. J. Microbiol. Methods 2000, 40, 175-179, doi:10.1016/S0167-7012(00)00122-6.

27. De la Fuente-Núñez, C.; Reffuveille, F.; Fernández, L.; Hancock, R.E.W. Bacterial biofilm strategies. Curr. Opin. Microbiol. 2013, 16, 580-589. 
bioRxiv preprint doi:https:/doi org/10.1101/2021.03 02 433687 this version posted March 3 2021. The copyright holder for this preprint (which was not lertified by peer review) is the author/funder, who has granted bioRxiv a license to display the preprint in perpetuity. It is made available under aCC-BY-NC-ND 4.0 International license.

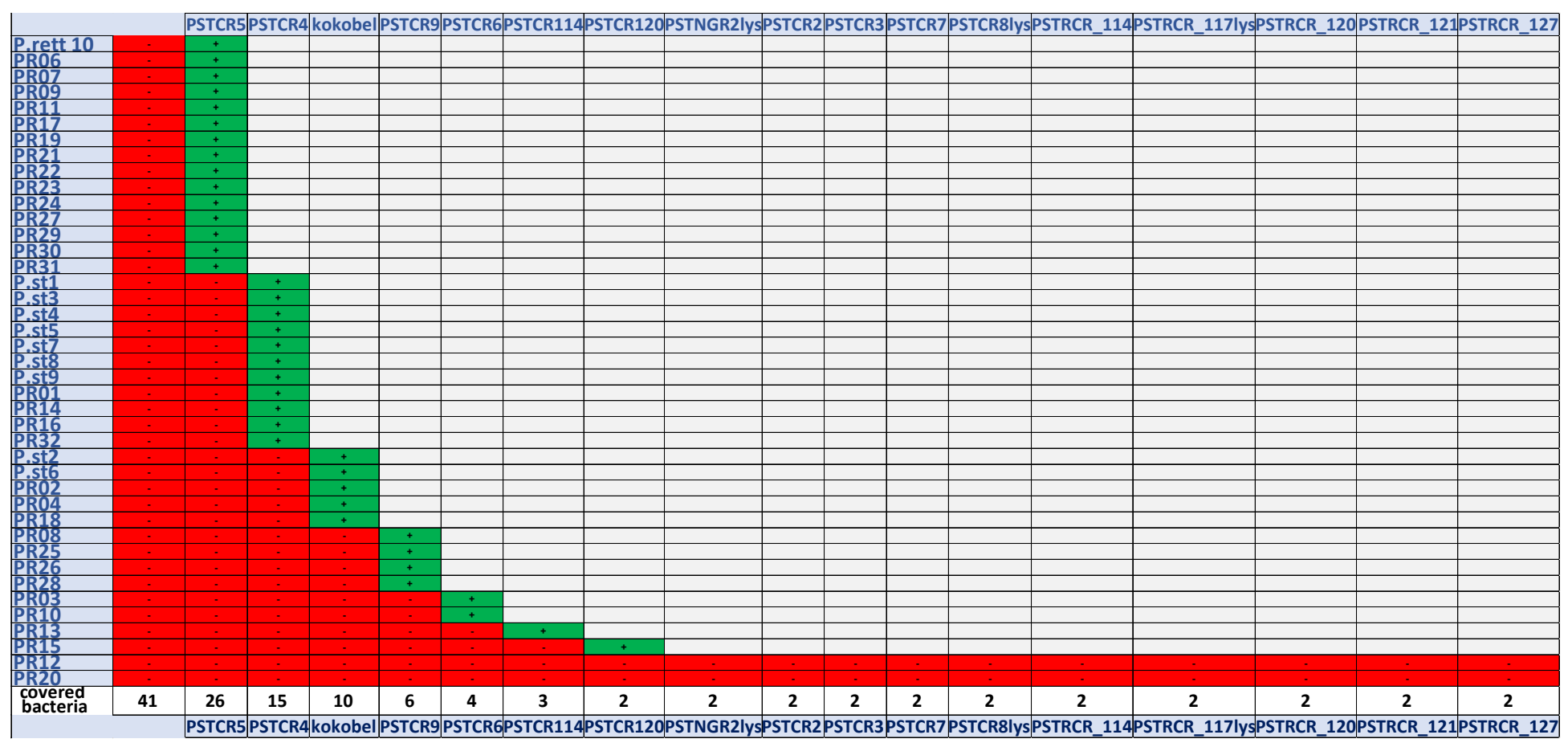


bioRxiv prepript.doi-https://doi.org/10.1101/2021.03.02.433687; this version posted March 3, 2021. The copyright holder for this preprint (which was not lertffied by peer review) is the author/funder, who has granted bioRxiv a license to display the preprint in perpetuity. It is made available under aCC-BY-NC-ND 4.0 International license.

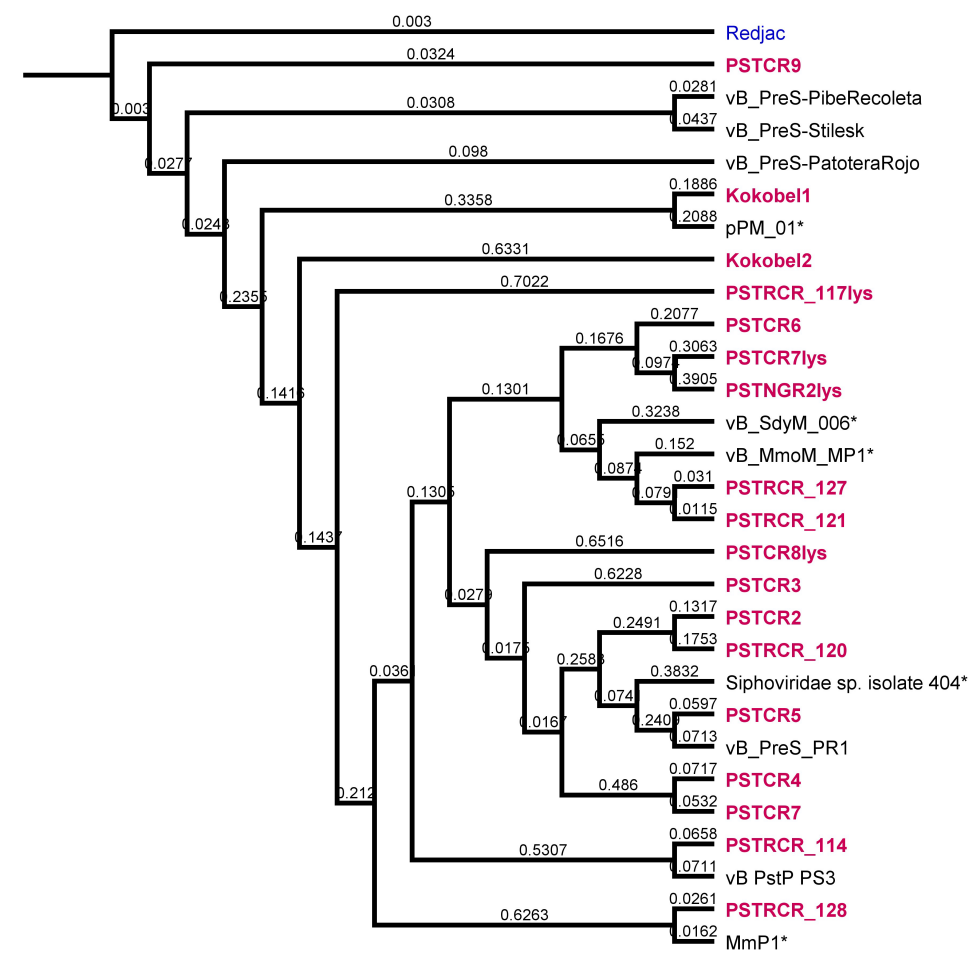


bioRxiv preprint doi: https://doi.org/10.1101/2021.03.02.433687; this version posted March 3, 2021. The copyright holder for this preprint (which was not.certified by peer review) is the author/funder, who has granted bioRxiv a license to display the preprint in perpetuity. It is Fig 4. made available under aCC-BY-NC-ND 4.0 International license.

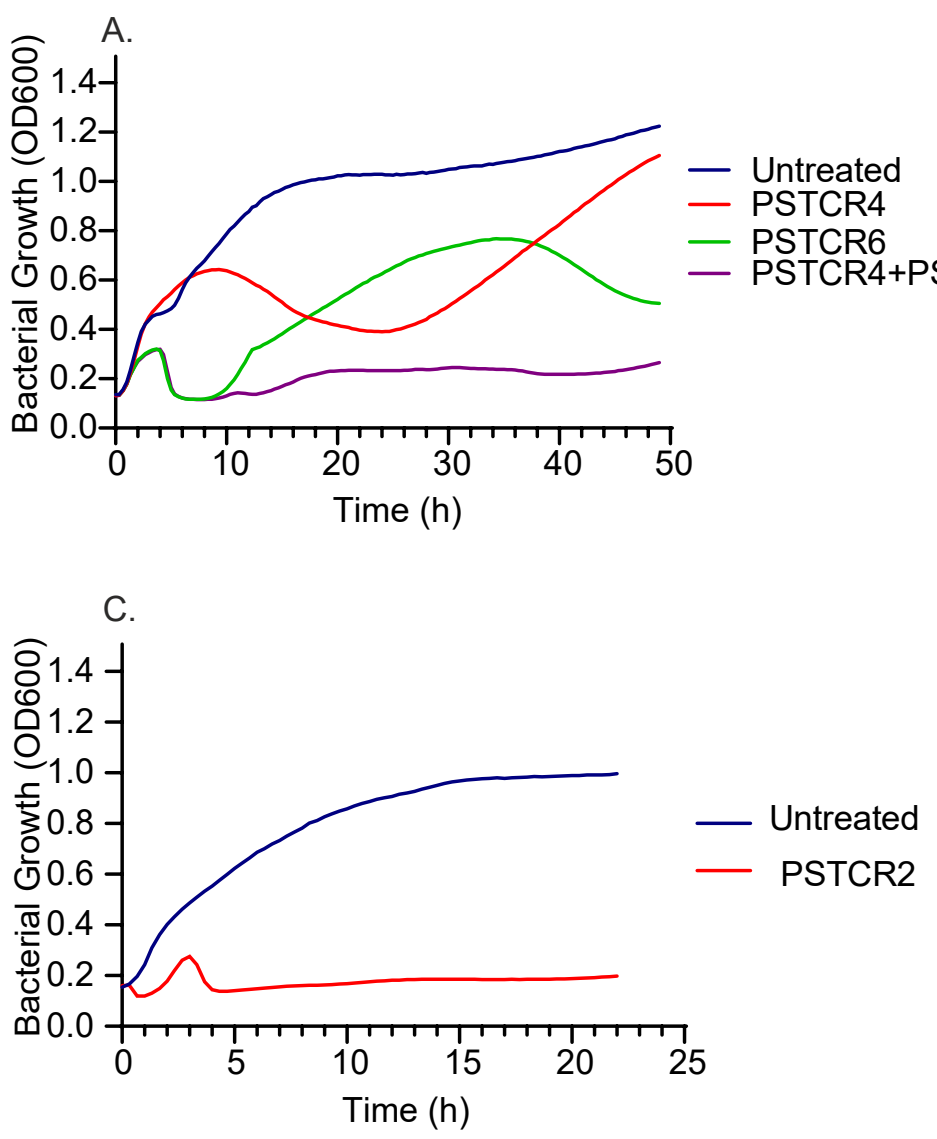

$B$.
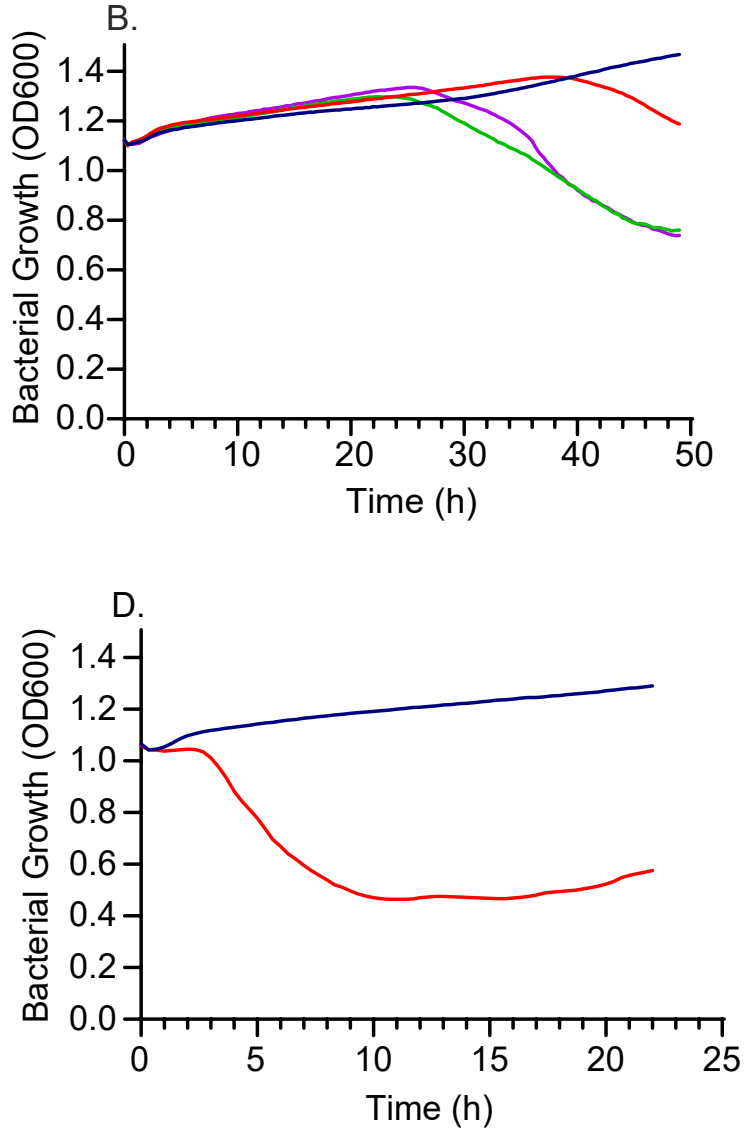
Fig 5.

A.

Rubber catheter

Silicon catheter
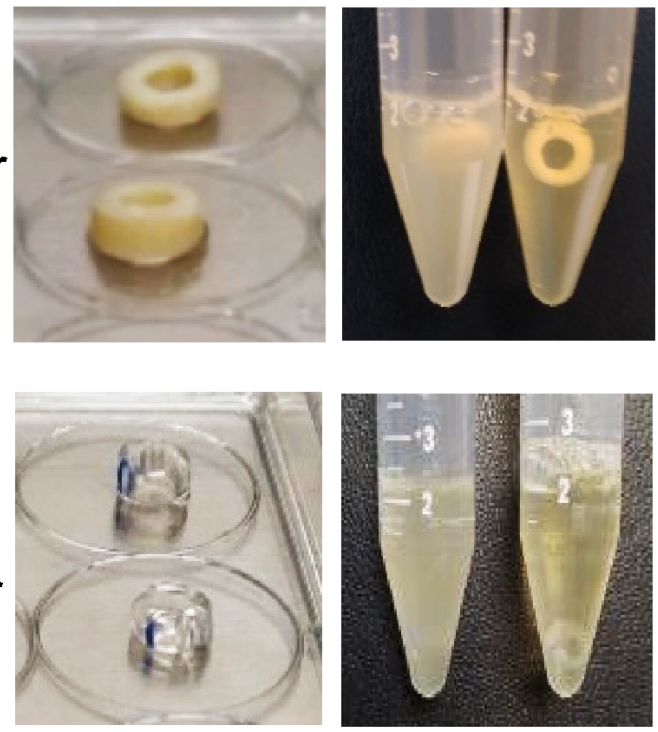

c.
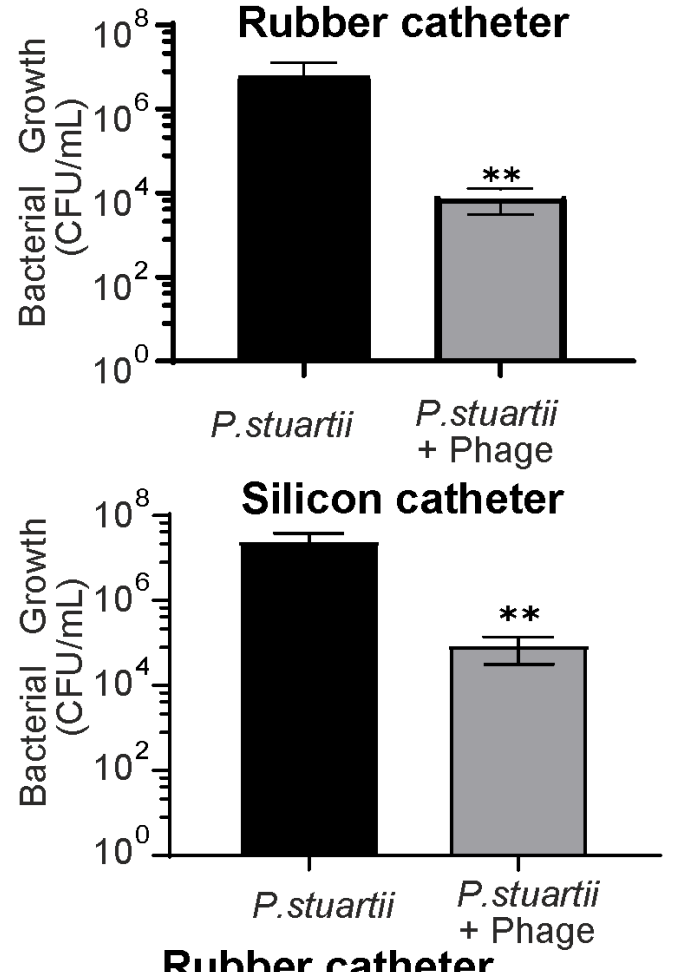

E. Rubber catheter
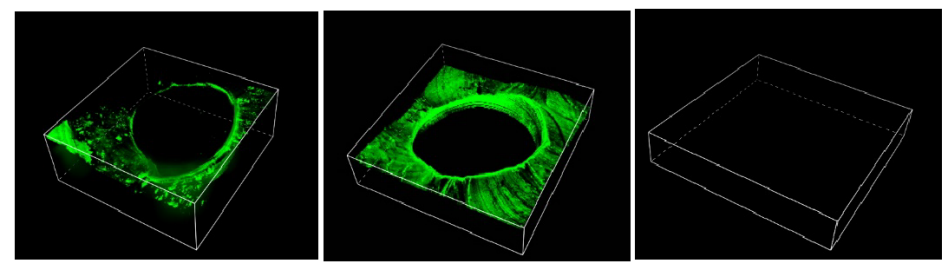

Untreated

$$
\text { P.stuartii }
$$

P.stuartii

+ Phage

Silicon catheter
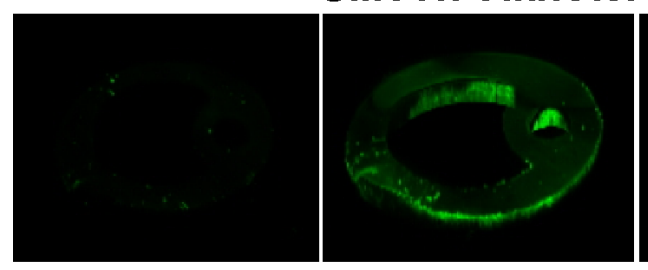

Untreated

P.stuartii

P.stuartii

+ Phage
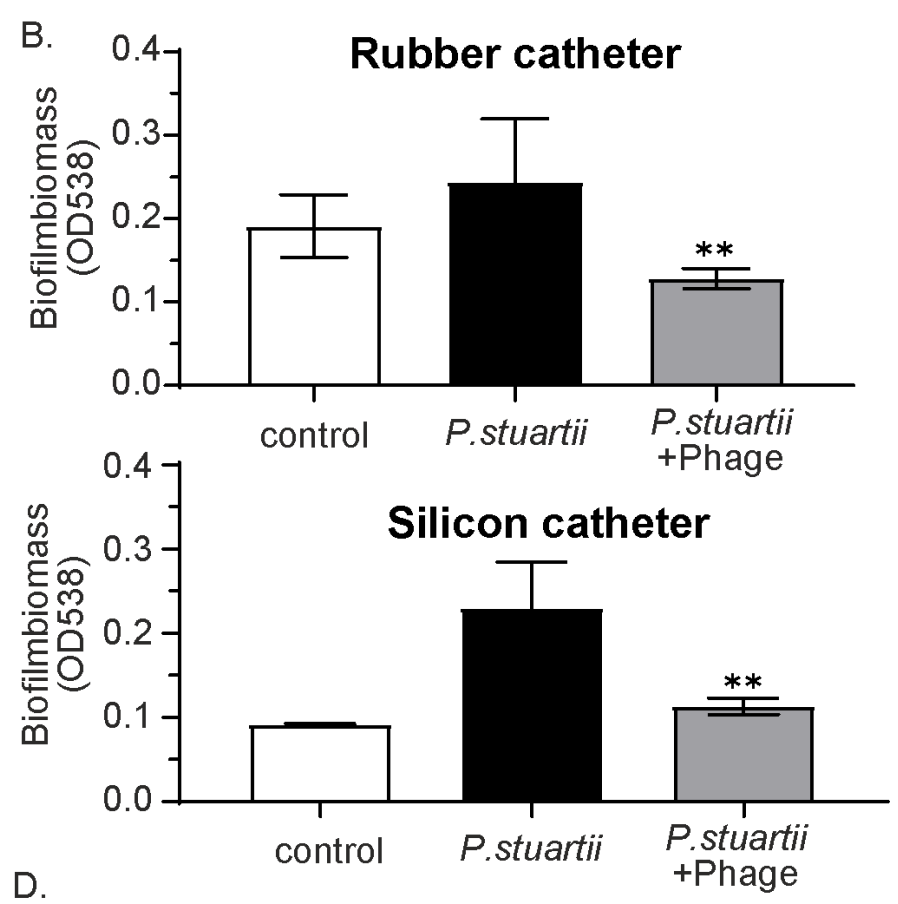

Rubber catheter

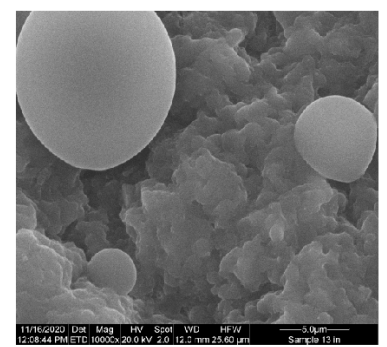

Untreated

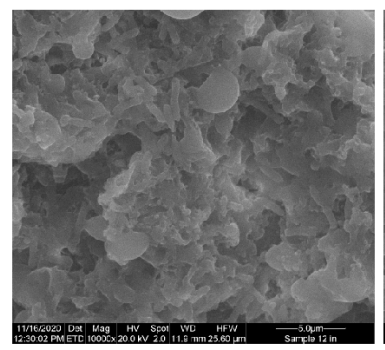

P. stuartii

Silicon catheter
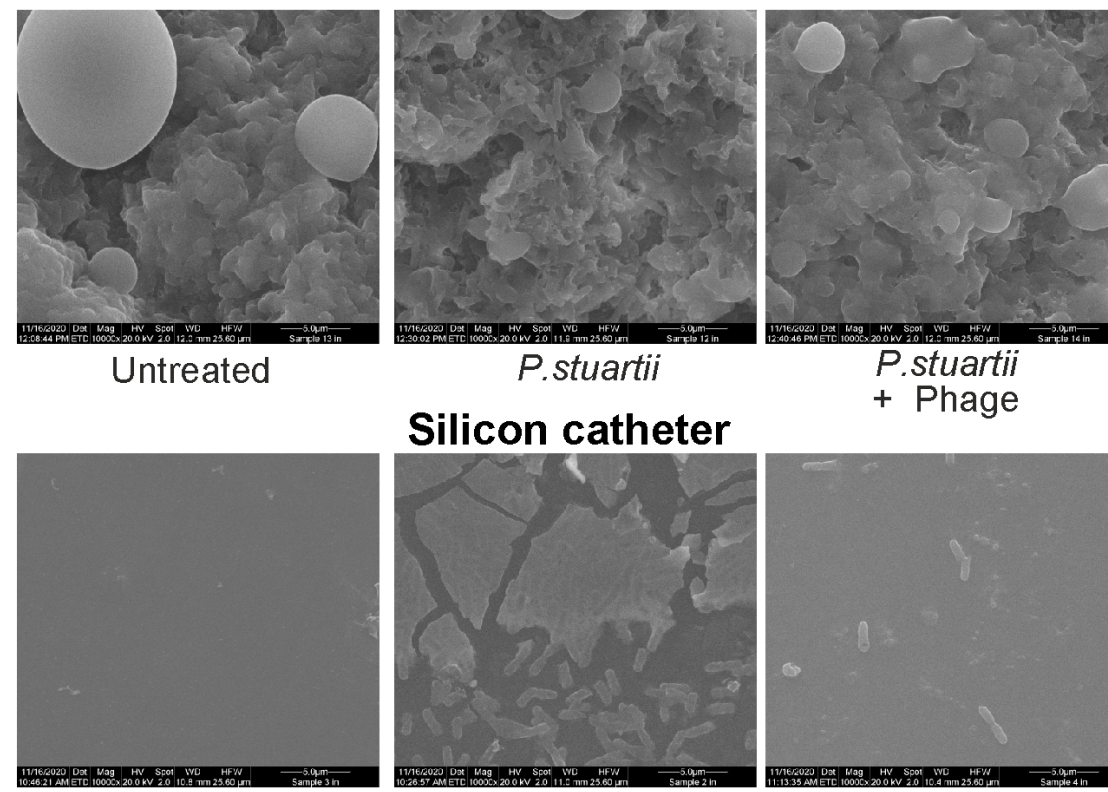

Untreated

P. stuartii

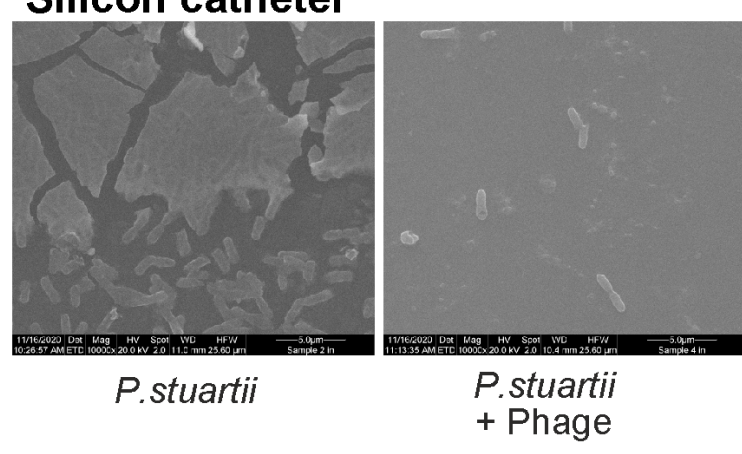

